Abstract P0-0102 Table 2 p values of pre- and post-operative variables in association with TIPSS failure

\begin{tabular}{|c|c|c|c|c|c|c|c|c|}
\hline \multirow{2}{*}{} & \multicolumn{3}{|c|}{ Successful TIPSS } & \multicolumn{3}{c|}{ Failed/Revised TIPSS } \\
\cline { 2 - 9 } & Pre-Operative & \multicolumn{2}{|c|}{ Post-operative } & \multicolumn{2}{|c|}{ Pre-operative } & \multicolumn{2}{c|}{ Post-operative } \\
\cline { 2 - 9 } & Mean & $\boldsymbol{P}$ value & Mean & $\boldsymbol{P}$ value & Mean & P value & Mean & $\boldsymbol{P}$ value \\
\hline Haemoglobin & 10.33 & 0.43 & 11.6 & 0.137 & 11.33 & 0.526 & 13.3 & 0.319 \\
\hline Platelets & 95.67 & 0.48 & 113 & 0.42 & 92.17 & 0.325 & 94.3 & 0.546 \\
\hline WBC & 9.33 & 0.318 & 4.62 & 0.474 & 5.83 & 0.158 & 4.6 & 0.614 \\
\hline ALT & 81.33 & 0.588 & 43.9 & 0.563 & 71.83 & 0.58 & 52.33 & 0.592 \\
\hline AST & 46 & 0.599 & 58.5 & 0.611 & 46.3 & 0.653 & 35.6 & 0.447 \\
\hline ALP & 145.33 & 0.105 & 275.5 & 0.615 & 372.17 & $0.027 *$ & 182.3 & 0.521 \\
\hline GGT & 22 & 0.426 & 56.7 & 0.316 & 36.5 & 0.451 & 105 & 0.354 \\
\hline PT & 12 & 0.505 & 16.9 & 0.582 & 15 & 0.392 & 18.7 & 0.681 \\
\hline PIT & 37.67 & 0.776 & 39.7 & 0.055 & 37.5 & 0.871 & 53.6 & 0.268 \\
\hline INR & 1.33 & 0.12 & 1.3 & 0.133 & 1.5 & 0.25 & 1.5 & 0.423 \\
\hline Urea & 3.33 & 0.656 & 3.9 & 0.634 & 3.33 & 0.653 & 4.38 & 0.607 \\
\hline Creatinine & 26 & 0.485 & 45.8 & 0.46 & 36.17 & 0.466 & 53.3 & 0.623 \\
\hline
\end{tabular}

Results A total of 10 children (7 females) underwent TIPSS. The primary indication was variceal haemorrhage in all the patients with different diagnoses (Table-1). Congenital Hepatic Fibrosis was the most common aetiology (50\%). Three patients $(30 \%)$ needed revision in a mean of 24.2 month post-procedure. 7 patients $(70 \%)$ did not require revision 36 month post procedure, with a mean follow up of 60.5 month.

Pre-operative high serum alkaline phosphatase (ALP) was associated with TIPSS failure ( $\mathrm{p}$ value $=0.027$ ) and thus, can be used as a pre-operative predictor for failed TIPSS. Other variables did not show significant association with TIPSS procedure including patients age and weight.

Conclusion This study involves the association of multiple variables with TIPSS outcomes in children. ALP showed a relative association as a predictor to TIPSS failure. Further studies are needed with a larger sample.

\section{PO-0103 EVALUATION OF THE PAEDIATRIC PATIENTS WITH GASTROINTESTINAL BLEEDING: EXPERIENCE OF A TERTIARY CENTRE}

T Ozkan, D Altay, A Otuzbir, T Ozgur. Pediatric Gastroenterology Hepatology and Nutrition, Uludag University Medical Faculty, Bursa, Turkey

\subsection{6/archdischild-2014-307384.772}

Introduction Gastrointestinal bleeding (GB) can be seen in children of all ages and it is one of the frequent application reason to paediatric gastroenterologists. Even though the causes of bleeding differs according to age groups, it may become lifethreatening depending on the severity of the bleeding.

Aim The aim of the study was to determine the demographical and etiological factors of patients who admitted to our clinic with upper or lower gastrointestinal bleeding.

Material and method 94 patients, were included to the study, admitted to Uludag University Faculty of Medicine Paediatric Gastroenterology department with upper or lower gastrointestinal bleeding between January 2010 and June 2013. Patients' files were evaluated retrospectively.

Results The number of patients with upper gastrointestinal bleeding (UGB) was 53, average ages of these patients was 11.1 years (2-18 years), $45.3 \%$ were female and $54.7 \%$ were male, respectively. At the aetiology of these bleeding cases H.pylori were detected in $18.8 \%$, peptic or duodenal ulcer were detected in 10 patients (range of ages 10-18 years, average 12 years) and H.pylori was detected in only two patients with ulcer. While all gastric mucosa were hyperemic in 26 patients, distal esophagitis or duodenitis were detected also in 23 patients with UGB.
Esophageal variceal bleeding was the cause of 4 patients with UGB and chronic renal failure was associated to bleeding in one patient. Barrett's oesophagus was detected histologically in 10 and 13 years of age two patients. Four patients had a history of nonsteroidal anti-inflammatory drug use prior to bleeding. Henoch-Schonlein disease was diagnosed in 7 years old male patient. Bleeding was the first symptom of this disease in this case and purpura was occurred after 4 days of bleeding. The remaining 41 cases were called idiopathic bleeding. Range of the ages of 41 patients with lower gastrointestinal bleeding (LGB) was 11.1 years and 24 patients were male $(58.5 \%$ male, $41.5 \%$ female, respectively). Ulcerative colitis was diagnosed histologically in 9 patients (21.9\%). Polip in rectum or sigmoid colon was detected in 6 patients, nonspesific chronic inflammation was reported pathologically in patients with polip and there were no family history for polip in these patients. Colonoscopy was normal in 13 patients (31.7\%) with LGB.

Conclusion Chronic gastritis was detected majority in aetiology of the patients with UGB and we thought that bad dietary habits had great importance in these cases. $21.9 \%$ cases with LGB were diagnosed with ulcerative colitis. All cases were evaluated, there were no death because of bleeding at the prognosis of these patients.

Discussion Gastrointestinal bleeding is one of important reason for reference of tertiary centre in children. Considering the frequency of H.pylori positivity in our society, $18.8 \%$ were found to be positive in cases with UGB. Although the vast majority of patients with LGB were idiopatic, ulcerative colitis plays an important role in patients with LGB.

\section{PO-0104 DIFFERENT CLINICAL SPECTRUM OF CYTOMEGALOVIRUS HEPATITIS IN INFANTS}

T Ozkan, D Altay, A Otuzbir, U Sahin, T Ozgur. Pediatric Gastroenterology Hepatology and Nutrition, Uludag University Medical Faculty, Bursa, Turkey

\subsection{6/archdischild-2014-307384.773}

Background and aim Cytomegalovirus (CMV) is, a member of the herpes viridae family, found widely in nature and the most common congenital infection in newborns. The average incidence of CMV infection in newborn infants is $1 \%$. Irreversible signs of central nervous system involvement (microcephaly, deafness, mental-motor retardation) develops in 5-10\% congenitally infected infants. Signs of perinatal infections, hepatosplenomegaly, pneumonia, hepatitis, are seen, but in this period neurological sequelae are rare. In this study, CMV-infected patients who were admitted to our clinic within 3 months were examined.

Methods Between the date of December 2013 and February 2014, in total five CMV infection ( $\min 45$ days, max 2 years-4 months old) have been detected at Uludag University, Faculty of Medicine, Department of Paediatric Gastroenterology. Three of those patients admitted with jaundice and other two patients were detected during the pancytopenia aetiology and vomiting aetiology investigation.

In our series, which consist of developed CMV hepatitis cases due to different etiological reasons, retrospective examination is conducted with clinical and laboratory findings.

Results Biliary atresia was detected in three patients. One is by intraoperative cholangiography and other two, as evidenced by histopathology. In the fourth case, CMV infection was detected simultaneously with vitamin $\mathrm{B}_{12}$ deficiency during pancytopenia investigation. In the fifth patient, performed liver transplantation due to neonatal hepatitis, CMV infection was detected during 